\title{
727.
}

\section{EQUATION OF THE WAVE-SURFACE IN ELLIPTIC COORDINATES.}

[From the Messenger of Mathematics, vol. viII. (1879), pp. 190, 191.]

THE equation of the wave-surface

$$
\frac{a x^{2}}{x^{2}+y^{2}+z^{2}-a}+\frac{b y^{2}}{x^{2}+y^{2}+z^{2}-b}+\frac{c z^{2}}{x^{2}+y^{2}+z^{2}}=0,
$$

when transformed to coordinates $p, q, r$, such that

$$
\begin{aligned}
& \frac{x^{2}}{-a+p}+\frac{y^{2}}{-b+p}+\frac{z^{2}}{-c+p}=1, \\
& \frac{x^{2}}{-a+q}+\frac{y^{2}}{-b+q}+\frac{z^{2}}{-c+q}=1, \\
& \frac{x^{2}}{-a+r}+\frac{y^{2}}{-b+r}+\frac{z^{2}}{-c+r}=1 ;
\end{aligned}
$$

(that is, to the elliptic coordinates belonging to the quadric surface $\frac{x^{2}}{-a}+\frac{y^{2}}{-b}+\frac{z^{2}}{-c}=1$ ), assumes the form

$$
(q+r-a-b-c)(r+p-a-b-c)(p+q-a-b-c)=0,
$$

(Senate-House Problem, January 14, 1879).

In fact, $p, q, r$ are the roots of the equation

$$
\frac{x^{2}}{-a+u}+\frac{y^{2}}{-b+u}+\frac{z^{2}}{-c+u}=1 ;
$$

we have therefore

$$
\begin{aligned}
(u-p)(u-q)(u-r) & =(u-a)(u-b)(u-c) \\
& -x^{2}(u-b)(u-c)-y^{2}(u-c)(u-a)-z^{2}(u-a)(u-b) ;
\end{aligned}
$$


whence, writing for shortness

we have

$$
\begin{array}{ll}
A=a+b+c, \quad P=p+q+r \\
B=b c+c a+a b, \quad Q=q r+r p+p q \\
C=a b c \quad, \quad R=p q r
\end{array}
$$

and thence also

$$
\begin{aligned}
x^{2}+\quad y^{2}+\quad z^{2} & =P-A, \\
(b+c) x^{2}+(c+a) y^{2}+(a+b) z^{2} & =Q-B, \\
b c x^{2}+\quad c a y^{2}+\quad a b z^{2} & =R-C,
\end{aligned}
$$

$$
\begin{aligned}
a(b+c) x^{2}+b(c+a) y^{2}+c(a+b) z^{2} & =B(P-A)-(R-C), \\
a x^{2}+\quad b y^{2}+\quad c z^{2} & =A(P-A)-(Q-B) .
\end{aligned}
$$

The equation of the wave-surface is

$$
a b c-\left\{a(b+c) x^{2}+b(c+a) y^{2}+c(a+b) z^{2}\right\}+\left(x^{2}+y^{2}+z^{2}\right)\left(a x^{2}+b y^{2}+c z^{2}\right)=0 .
$$

By the formulæ just obtained, this is

that is,

$$
C-[B(P-A)-(R-C)]+(P-A)[A(P-A)-(Q-B)]=0,
$$

that is,

$$
A^{3}-2 A^{2} P+A\left(P^{2}+Q\right)-(P Q-R)=0,
$$

$$
\{A-(q+r)\}\{A-(r+p)\}\{A-(p+q)=0,
$$

or, substituting for $A$ its value $a+b+c$, and reversing the sign of each factor, we have the formula in question.

It is easy to see that, taking $a, b, c$ to be each positive, $(a>b>c)$, and assuming also $p>q>r$, we obtain the different real points of space by giving to these coordinates respectively the different real values from $\infty$ to $a, a$ to $b$, and $b$ to $c$ respectively. Hence

$\begin{array}{ccc} & \text { greatest, } & \text { least value, is } \\ q+r, & a+b, & a+c, \\ r+p, & \infty, & a+c, \\ p+q, & \infty, & a+b,\end{array}$

so that $r+p, p+q$, may be either of them $=a+b+c$, but $q+r$ cannot be $=a+b+c$, that is, $q+r=a+b+c$ does not belong to any real point on the wave-surface. We can only have $r+p$ and $p+q$ each $=a+b+c$, if $p=a+c, q=r=b$, and these values belong as is easily shown to the nodes on the wave-surface; hence, the equations $r+p=a+b+c$ and $p+q=a+b+c$ being satisfied simultaneously only at the nodes of the surface, must belong to the two sheets respectively. And it can be shown that $p+r=a+b+c$ belongs to the external sheet, and $p+q=a+b+c$ belongs to the internal sheet. In fact, for the point $(0,0, \sqrt{ } a)$, which is on the external sheet, we have $p=a+c, q=a, r=b$, and therefore $p+r=a+b+c$ : for the point $(0,0, \sqrt{ } b)$, which is on the internal sheet, either

$$
(p=b+c, q=a, r=b) \quad \text { or } \quad(p=a, q=b+c, r=c),
$$

according as $b+c>a$ or $b+c<a$ : but in each case

$$
p+q=a+b+c \text {. }
$$

